\title{
On the performance of economic model predictive control with self-tuning terminal cost ${ }^{\text {th }}$
}

\author{
Matthias A. Müller ${ }^{\mathrm{a}, 1}$, David Angeli ${ }^{\mathrm{b}, \mathrm{c}}$, Frank Allgöwera,1 \\ ${ }^{a}$ Institute for Systems Theory and Automatic Control, University of Stuttgart, 70550 Stuttgart, Germany. \\ ${ }^{b}$ Department of Electrical and Electronic Engineering, Imperial College, London, UK. \\ ${ }^{c}$ Dipartimento Sistemi e Informatica, University of Florence, Italy.
}

\begin{abstract}
In this paper, we analyze the closed-loop performance of a recently introduced economic model predictive control (MPC) scheme with self-tuning terminal cost. To this end, we propose to use a generalized terminal region constraint instead of a generalized terminal equality constraint within the repeatedly solved optimization problem, which allows us to obtain improved closed-loop asymptotic average performance bounds. In particular, these bounds can be obtained a priori. We discuss how the necessary parameters for the generalized terminal region setting can be calculated, and we illustrate our findings with two numerical examples.

Keywords: Economic model predictive control, Self-tuning terminal cost, Generalized terminal constraint
\end{abstract}

\section{Introduction}

In recent years, a variant of model predictive control (MPC) termed economic $M P C$ has received an increasing amount of attention. In contrast to standard tracking (or stabilizing) MPC, the primary control objective in economic MPC is not the stabilization of a given setpoint (or trajectory to be tracked), but rather the optimization of a given general performance criterion, possibly related the economics of the considered process. On a technical level, this means that the cost function in economic MPC needs not be positive definite with respect to some setpoint, as is typically assumed

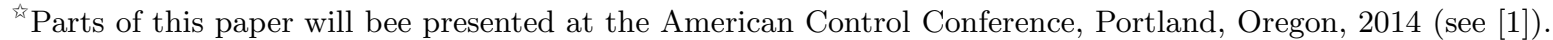

Email addresses: matthias.mueller@ist.uni-stuttgart.de (Matthias A. Müller), d.angeli@imperial.ac.uk (David Angeli), frank.allgower@ist.uni-stuttgart.de (Frank Allgöwer)

${ }^{1}$ Supported by the German Research Foundation (DFG) within the Priority Programme 1305 "Control Theory of Digitally Networked Dynamical Systems" and within the Cluster of Excellence in Simulation Technology (EXC 310/1) at the University of Stuttgart.
} 
in standard tracking MPC. In the literature, various properties of economic MPC such as average performance and convergence of the resulting closed-loop system, optimal steady-state operation and fulfillment of average constraints were studied using different assumptions and/or additional (terminal) constraints (see, e.g., $[2,3,4,5,6,7]$ ). Furthermore, various applications of economic MPC have recently been reported, such as the ones in $[8,9,10,11]$.

In this paper, we study an MPC framework using a generalized terminal constraint, meaning that the endpoint of the predicted state sequence has to be equal to some arbitrary steady-state (or contained in a terminal region around an arbitrary steady-state) and not to a specific one. Such a generalized terminal constraint setting has first been proposed in the context of tracking MPC $[12,13]$, and recently also in economic MPC $[14,15,16]$. The main benefits compared to a setting with fixed terminal point or terminal region constraint are a possibly much larger region of attraction and a guarantee of recursive feasibility even in case that the cost function (and hence also the optimal steady-state) changes online. Furthermore, in the context of economic MPC, a priori knowledge of the optimal steady-state is not required in $[15,16]$, which is needed when using a fixed terminal constraint.

On the other hand, a disadvantage of using a generalized terminal constraint in economic MPC is that closed-loop performance guarantees are not as easily obtained as in case of a fixed terminal constraint. In particular, in [15], for general initial conditions closed-loop performance bounds are only obtained under an additional controllability assumption and by overriding the MPC algorithm, i.e., if necessary, following the previously optimal solution. In [16], an economic MPC algorithm with self-tuning terminal cost was proposed, inspired by the one in [15] with fixed terminal weight. As was shown in [16], the benefits of a self-tuning terminal weight compared to a fixed one are (i) that the terminal weight can possibly be kept much smaller, which can be good both for numerical and (closed-loop) performance reasons, and (ii) the possibility to obtain closed-loop average performance bounds without further controllability assumptions and without possibly overriding the MPC algorithm as done in [15, Algorithm 3]. However, the resulting average performance bounds obtained in [16] are rather of conceptual nature, in the sense that they can only be verified a posteriori. Namely, the closed-loop system outperforms in average the cost of the best steady-state achievable from the $\omega$-limit set of the resulting closed-loop trajectory (see Section 2.3 for further details). This cannot, in general, be determined a priori. 
The contribution of this paper is to develop an economic MPC scheme with generalized terminal constraint and self-tuning terminal cost for which improved and a priori verifiable bounds on the closed-loop asymptotic average performance can be obtained. To this end, we modify the scheme proposed in [16] and replace the generalized terminal equality constraint by a generalized terminal region constraint; this idea has also been used in the context of tracking MPC (see, e.g., [12, 13]). This allows us to show that the closed-loop average performance is at least as good as a value corresponding to a local minimum of the stage cost function restricted to the set of feasible steadystates. For linear systems with convex cost and constraints, this results in the average performance being at least as good as the optimal steady-state, which recovers results obtained for a fixed terminal constraint $[2,3]$.

The remainder of this paper is organized as follows. In Section 2, we introduce the proposed economic MPC scheme with self-tuning terminal cost and generalized terminal region constraint; furthermore, we briefly review the results obtained in [16] and show that they carry over to the modified setting considered in this paper. The main results of this paper concerning improved and a priori verifiable bounds for the closed-loop average performance are then given in Section 3. In Section 4 , we discuss how the necessary parameters for the generalized terminal region setting can be calculated. We illustrate our findings with two numerical examples in Section 5, before concluding the paper in Section 6. We close this section by noting that parts of the results presented in this paper have also appeared in the conference version [1]. The main novelties of this paper compared to [1] are (i) that the complete proof of our main result is included in Section 3, (ii) the design procedure of the terminal ingredients for nonlinear systems (Section 4.2) and (iii) the numerical examples in Section 5 illustrating our results.

\subsection{Notation}

Let $\mathbb{I}_{\geq 0}$ denote the set of nonnegative integers, and $\mathbb{I}_{[a, b]}$ the set of all integers in the interval $[a, b] \subseteq \mathbb{R}$. We define $B_{\varepsilon}(y)$ to be the ball of radius $\varepsilon>0$ around the point $y \in \mathbb{R}^{n}$, i.e., $B_{\varepsilon}(y):=$ $\left\{x \in \mathbb{R}^{n}:|x-y| \leq \varepsilon\right\}$. For a function $g: \mathbb{R}^{n} \rightarrow \mathbb{R}, g_{x}(y)$ denotes the gradient and $g_{x x}(y)$ the Hessian of $g$ with respect to $x$, evaluated at the point $y \in \mathbb{R}^{n}$. Given two sets $\mathcal{A}, \mathcal{B} \subseteq \mathbb{R}^{n}$, the Minkowski set addition and Pontryagin set difference are defined as $\mathcal{A} \oplus \mathcal{B}:=\{a+b: a \in \mathcal{A}, b \in \mathcal{B}\}$ and $\mathcal{A} \ominus \mathcal{B}:=\{a \in \mathcal{A}: a+b \in \mathcal{A} \forall b \in \mathcal{B}\}$, respectively. For a symmetric matrix $A \in \mathbb{R}^{n \times n}$, denote by $\lambda_{\min }(A)$ and $\lambda_{\max }(A)$ its minimum and maximum eigenvalue, respectively. 


\section{Economic MPC with self-tuning terminal cost}

We consider discrete-time nonlinear systems of the form

$$
x(t+1)=f(x(t), u(t)), \quad x(0)=x_{0},
$$

with $x(t) \in \mathbb{X} \subseteq \mathbb{R}^{n}$ and $u(t) \in \mathbb{U} \subseteq \mathbb{R}^{m}$ for all $t \in \mathbb{I}_{\geq 0}$, and $f: \mathbb{R}^{n} \times \mathbb{R}^{m} \rightarrow \mathbb{R}^{n}$ is assumed to be continuous. The system is subject to (possibly coupled) pointwise-in-time state and input constraints $(x(t), u(t)) \in \mathbb{Z}$ for all $t \in \mathbb{I}_{\geq 0}$, where $\mathbb{Z} \subseteq \mathbb{X} \times \mathbb{U}$ is assumed to be compact. Denote by $\mathbb{Z}_{\mathbb{X}}$ the projection of $\mathbb{Z}$ on $\mathbb{X}$. The control objective is to design a control law such that the resulting closed-loop system satisfies the given state and input constraints and such that a desired objective function $\ell$ is minimized. Here, the stage cost $\ell: \mathbb{X} \times \mathbb{U} \rightarrow \mathbb{R}$ is assumed to be continuous, but can otherwise be an arbitrary, possibly economic, function which need not satisfy any convexity or definiteness assumption. Furthermore, by $\left(x_{s}, u_{s}\right)$ we denote an optimal steady-state achieving the minimal cost of all steady-states in the set $\mathbb{Z}$, i.e., $\left(x_{s}, u_{s}\right)$ satisfies

$$
\ell\left(x_{s}, u_{s}\right)=\min _{(x, u) \in \mathbb{Z}, x=f(x, u)} \ell(x, u) .
$$

Note that as $\ell$ is continuous and $\mathbb{Z}$ is compact, we can assume without loss of generality that $\ell\left(x_{s}, u_{s}\right)=0$.

\subsection{Generalized terminal state constraint}

For the setting as described above, in [16] we proposed the following economic MPC scheme with a self-tuning terminal cost, which we briefly recall in the following for the sake of completeness; this is a variation of the one introduced in [15] with fixed terminal weight. Namely, at each time $t$ with $x:=x(t)$, the following optimization problem is solved:

$$
\min _{u(0 \mid t), \ldots, u(N \mid t)} \sum_{k=0}^{N-1} \ell(x(k \mid t), u(k \mid t))+\beta(t) \ell(x(N \mid t), u(N \mid t))
$$

subject to

$$
\begin{aligned}
x(0 \mid t) & =x \\
x(k+1 \mid t) & =f(x(k \mid t), u(k \mid t)) \quad k \in \mathbb{I}_{[0, N-1]} \\
(x(k \mid t), u(k \mid t)) & \in \mathbb{Z}, \quad k \in \mathbb{I}_{[0, N]} \\
x(N \mid t) & =f(x(N \mid t), u(N \mid t)), \\
\ell(x(N \mid t), u(N \mid t)) & \leq \kappa(t),
\end{aligned}
$$


for some possibly time-varying terminal weight $\beta$ and $\kappa$ specified later. The notation $x(\cdot \mid t)$ and $u(\cdot \mid t)$ denote predicted state and input values (predicted at time $t$ ), respectively. As already discussed in the introduction, the main advantages of using a generalized terminal state constraint (4d) instead of a fixed terminal point constraint lie in the fact that a possibly much larger region of attraction is obtained, and that the optimal steady-state $\left(x_{s}, u_{s}\right)$, which is normally used as a fixed terminal point constraint [2], does not have to be known a priori.

\subsection{Generalized terminal region constraint}

In this paper we propose a relaxed form of the MPC algorithm (3)-(4). Namely, instead of requiring the terminal predicted state to be equal to some steady-state as in (4d), we require the terminal predicted state to lie in a terminal region $\mathbb{X}^{f}(\bar{x})$ around some steady-state $\bar{x}$. This leads to the following optimization problem to be solved at each time instant $t$ with $x:=x(t)$ :

$$
\min _{u(0 \mid t), \ldots, u(N-1 \mid t), \bar{x}(t), \bar{u}(t)} \sum_{k=0}^{N-1} \ell(x(k \mid t), u(k \mid t))+V_{f}(x(N \mid t), \bar{x}(t))+\beta(t) \ell(\bar{x}(t), \bar{u}(t))
$$

subject to

$$
\begin{aligned}
x(0 \mid t) & =x \\
x(k+1 \mid t) & =f(x(k \mid t), u(k \mid t)) \quad k \in \mathbb{I}_{[0, N-1]} \\
(x(k \mid t), u(k \mid t)) & \in \mathbb{Z}, \quad k \in \mathbb{I}_{[0, N-1]} \\
x(N \mid t) & \in \mathbb{X}^{f}(\bar{x}(t)), \quad \bar{x}(t)=f(\bar{x}(t), \bar{u}(t)) \\
(\bar{x}(t), \bar{u}(t)) & \in \overline{\mathbb{Z}} \subseteq \mathbb{Z} \\
\ell(\bar{x}(t), \bar{u}(t)) & \leq \kappa(t) .
\end{aligned}
$$

Denote the optimal solution to problem (5)-(6) by ${ }^{2} \bar{x}^{*}(t), \bar{u}^{*}(t)$ and $\mathbf{u}^{*}(t):=\left\{u^{*}(0 \mid t), \ldots, u^{*}(N-\right.$ $1 \mid t)\}$ and the corresponding state sequence by $\mathbf{x}^{*}(t):=\left\{x^{*}(0 \mid t), \ldots, x^{*}(N \mid t)\right\}$; furthermore, the optimal value function is denoted by $V(x, \beta, \kappa)$. As usual in MPC, the first part of the optimal input sequence, $u^{*}(0 \mid t)$, is applied to the system (1) at time $t$. The parameter $\kappa$ is updated according to the cost of the previous optimal steady-state around which the terminal region was built, i.e.,

\footnotetext{
${ }^{2}$ For simplicity, we assume that $\mathbf{u}^{*}(t), \bar{x}^{*}(t), \bar{u}^{*}(t)$ are unique. If this is not the case, just assign a unique constant selection map to select one of the multiple minima.
} 
the following closed-loop system is obtained:

$$
\begin{aligned}
x(t+1) & =f\left(x(t), u^{*}(0 \mid t)\right), & x(0) & =x_{0}, \\
\kappa(t+1) & =\ell\left(\bar{x}^{*}(t), \bar{u}^{*}(t)\right), & \kappa(0) & =\kappa_{0} \geq 0 .
\end{aligned}
$$

Finally, the terminal weight $\beta$ follows some general causal update rule $B$ :

$$
\beta(t+1)=B(\beta(.), x(.), \kappa(.)), \quad \beta(0)=\beta_{0} \geq 0 .
$$

The interested reader is referred to [16] for different specific update rules $B$ and a detailed discussion regarding initialization (of $\kappa$ and $\beta$ ) and implementation issues.

Remark 1. As pointed out before, the constraints (6d)-(6f) are a generalization of $(4 \mathrm{~d})-(4 \mathrm{e})$. In fact, if a generalized terminal equality constraint is used, i.e., $\mathbb{X}^{f}(\bar{x}):=\{\bar{x}\}$, then (6d) and (6f) reduce to $(4 \mathrm{~d})-(4 \mathrm{e}) ;$ furthermore, in this case we can choose $\overline{\mathbb{Z}}=\mathbb{Z}$, and hence (6e) reduces to the the last constraint of (4c), i.e., for $k=N$ (see below the definition of the set $\overline{\mathbb{Z}}$ and a further discussion of the constraint (6e)). Also, without loss of generality the terminal cost $V_{f}$ satisfies $V_{f}(\bar{x}, \bar{x})=0$, and hence the cost function (5) reduces to (3) in case that $\mathbb{X}^{f}(\bar{x}):=\{\bar{x}\}$.

We make the following assumption on the set $\overline{\mathbb{Z}}$ (which is supposed to be compact), the terminal region $\mathbb{X}^{f}$ and the terminal cost $V_{f}$.

Assumption 1. Let $\alpha>0$ and $P, Q>0$. For each steady-state $(\bar{x}, \bar{u}) \in \overline{\mathbb{Z}}$, there exists a terminal region of the form $\mathbb{X}^{f}(\bar{x}):=\left\{x \in \mathbb{R}^{n}: E(x, \bar{x}) \leq \alpha\right\}$ with $E(x, \bar{x}):=(x-\bar{x})^{T} P(x-\bar{x})$, a continuous auxiliary terminal control law $\kappa_{f}(x, \bar{x})$ with $\kappa_{f}(\bar{x}, \bar{x})=\bar{u}$, and a continuous terminal cost function $V_{f}(x, \bar{x})$ such that the following is satisfied for all $x \in \mathbb{X}^{f}(\bar{x})$ :

(i) $\left(x, \kappa_{f}(x, \bar{x})\right) \in \mathbb{Z}$,

(ii) $E\left(f\left(x, \kappa_{f}(x, \bar{x})\right), \bar{x}\right)-E(x, \bar{x}) \leq-(x-\bar{x})^{T} Q(x-\bar{x})$,

(iii) $V_{f}\left(f\left(x, \kappa_{f}(x, \bar{x})\right), \bar{x}\right)-V_{f}(x, \bar{x}) \leq-\ell\left(x, \kappa_{f}(x, \bar{x})\right)+\ell(\bar{x}, \bar{u})$.

Remark 2. For a fixed $\bar{x}$, conditions (i)-(iii) of Assumption 1 reduce to standard conditions imposed when using a terminal cost/region framework, both in the case of tracking and economic $M P C[17,3]$. In (ii), we require something slightly stronger than invariance of the terminal region, 
namely that the terminal region is contractive if the local controller is applied; this is crucial for our main results later on. Note that it is sufficient if this holds for some arbitrary positive definite $Q$. We will discuss in Section 4 how Assumption 1 can be satisfied for all $(\bar{x}, \bar{u}) \in \overline{\mathbb{Z}}$.

The set $\overline{\mathbb{Z}} \subseteq \mathbb{Z}$ in (6e) has to be defined such that condition $(i)$ in Assumption 1 can be satisfied for all steady-states $(\bar{x}, \bar{u}) \in \overline{\mathbb{Z}}$, i.e., such that state and input constraints are satisfied in the terminal region around each steady-state $(\bar{x}, \bar{u}) \in \overline{\mathbb{Z}}$. In general, $\overline{\mathbb{Z}}$ depends on the size of the terminal region, i.e., on $\alpha$ (for given $P$ ). Namely, the larger $\alpha$, the smaller $\overline{\mathbb{Z}}$ has to be. In Section 4 , we show how both the terminal ingredients as well as $\overline{\mathbb{Z}}$ can be defined such that Assumption 1 is satisfied. There, we also further discuss the constraint (6e) and how the feasible sets of problems (3)-(4) and (5)-(6) are related. Moreover, instead of using a fixed $\alpha$, i.e., a fixed size of the terminal regions, one could also use state- or time-dependent $\alpha$, in order to obtain a larger set $\overline{\mathbb{Z}}$. This will be discussed in more detail in Remark 5 in Section 4. Finally, as already pointed out in the Introduction, we note that the idea of a generalized terminal region constraint was already used in the context of standard tracking MPC (see, e.g., [12, 13]), defined however in a different way. In this respect, in Section 4 we also comment on how the generalized terminal region constraint used in problem (5)-(6) relates to the so-called "invariant set for tracking" as used in [12, 13].

\subsection{Conceptual performance bounds}

In this section, we briefly review the results obtained in [16] and show that they carry over to the modified setting with a generalized terminal region. To this end, define the set of steady-states $(\bar{x}, \bar{u})$ such that a terminal region (as specified in Assumption 1) around $\bar{x}$ is reachable in $N>0$ steps from a point $y \in \mathbb{Z}_{\mathbb{X}}$ as

$$
\begin{aligned}
\mathcal{X}_{N}(y):=\left\{(\bar{x}, \bar{u}) \in \overline{\mathbb{Z}}: \exists \mathbf{u} \in \mathbb{U}^{N} \text { s.t. } x(0)=y, x(j+1)=f(x(j), u(j)) \forall j \in \mathbb{I}_{[0, N-1]},\right. \\
\left.(x(j), u(j)) \in \mathbb{Z} \forall j \in \mathbb{I}_{[0, N-1]}, x(N) \in \mathbb{X}^{f}(\bar{x}), \bar{x}=f(\bar{x}, \bar{u})\right\} .
\end{aligned}
$$

Note that for each $y \in \mathbb{Z}_{\mathbb{X}}$, the set $\mathcal{X}_{N}(y)$ is compact as $\mathbb{Z}, \overline{\mathbb{Z}}$ and the terminal regions $\mathbb{X}^{f}(\bar{x})$ are compact and $f$ is continuous. We now define the best achievable steady-state cost from a 
point $y \in \mathbb{Z}_{\mathbb{X}}$ as $^{3}$

$$
\begin{aligned}
& \ell_{\min }(y):=\min _{x, u} \ell(x, u) \\
& \text { s.t. }(x, u) \in \mathcal{X}_{N}(y)
\end{aligned}
$$

Furthermore, we define the best robustly achievable steady-state cost from a point $y \in \mathbb{Z}_{\mathbb{X}}$ as follows. For each $\varepsilon \geq 0$, let

$$
\ell_{\min }(y, \varepsilon):=\sup _{z \in B_{\varepsilon}(y) \cap \mathbb{Z}_{\mathbb{X}}} \ell_{\min }(z)
$$

denote the supremum of the best achievable steady-state cost on the set $B_{\varepsilon}(y) \cap \mathbb{Z}_{\mathbb{X}}$. With this, we define the best robustly achievable steady-state cost from a point $y \in \mathbb{Z}_{\mathbb{X}}$ as

$$
\bar{\ell}_{\min }(y):=\lim _{\varepsilon \searrow 0} \ell_{\min }(y, \varepsilon)
$$

Note that the limit in (12) exists as $\ell_{\min }(y, \varepsilon)$ is monotonically nonincreasing as $\varepsilon \searrow 0$. From the definitions in (10) and (12), it immediately follows that for each $y \in \mathbb{Z}_{\mathbb{X}}$ we have $\ell_{\min }(y) \leq \bar{\ell}_{\min }(y)$; however, equality does not hold in general as $\ell_{\min }(y, \varepsilon)$ is not necessarily continuous in $\varepsilon$ at $\varepsilon=0$ (see [16, Example 4] for a simple illustration of this fact).

Given the above, we can now recap the average performance results from [16] for the closedloop system (7). To this end, note that from (6f) and (7), it follows that the sequence $\kappa(t)$ is nonincreasing; as it is also bounded from below (by $\ell\left(x_{s}, u_{s}\right)=0$ ), it converges and we denote its limit by $\kappa_{\infty}$. Furthermore, we make appropriate assumptions on the closed-loop terminal weight sequence $\beta(\cdot)$ resulting from (7)-(8). To this end, let $\gamma(t):=\beta(t+1)-\beta(t)$.

Assumption 2. The sequence $\beta(\cdot)$ satisfies $\gamma(t) \leq c$ and $\beta(t) \geq \underline{\beta}$ for all $t \in \mathbb{I}_{\geq 0}$ and some constants $c, \underline{\beta} \in \mathbb{R}$, and $\lim \sup _{t \rightarrow \infty} \gamma(t) \leq 0$.

Assumption 3. The sequence $\beta(\cdot)$ satisfies $\gamma(t) \leq c$ and $\beta(t) \geq \underline{\beta}$ for all $t \in \mathbb{I}_{\geq 0}$ and some constants $c, \underline{\beta} \in \mathbb{R}$, and $\liminf _{t \rightarrow \infty} \beta(t)<\infty$.

Theorem 1 ([16, Theorem 1]). Suppose that Assumption 1 is satisfied and the optimization problem (5)-(6) is feasible at $t=0$. Then it is feasible for all $t \in \mathbb{I}_{\geq 0}$. Consider the closed-loop

\footnotetext{
${ }^{3}$ In the following, if a minimum is taken over the empty set, then by convention the minimum is $+\infty$.
} 
system (7)-(8). If $\beta(\cdot)$ satisfies Assumption 2, then

$$
\limsup _{T \rightarrow \infty} \frac{\sum_{t=0}^{T-1} \ell(x(t), u(t))}{T} \leq \kappa_{\infty}
$$

If $\beta(\cdot)$ satisfies Assumption 3, then

$$
\liminf _{T \rightarrow \infty} \frac{\sum_{t=0}^{T-1} \ell(x(t), u(t))}{T} \leq \kappa_{\infty}
$$

Proof: As usual in MPC, a feasible solution to problem (5)-(6) at time $t+1$ is given by the end piece of the previously optimal solution appended by the terminal controller, i.e., $\hat{\mathbf{u}}(t+1):=$ $\left\{u^{*}(1 \mid t), \ldots, \kappa_{f}\left(x^{*}(N \mid t), \bar{x}^{*}(t)\right)\right\}$, and $(\bar{x}(t+1), \bar{u}(t+1)):=\left(\bar{x}^{*}(t), \bar{u}^{*}(t)\right)$. This means that the optimization problem (5)-(6) is recursively feasible. With this, we obtain

$$
\begin{aligned}
& V(x(t+1), \beta(t+1), \kappa(t+1))-V(x(t), \beta(t), \kappa(t)) \\
& \leq-\ell(x(t), u(t))+\ell\left(x^{*}(N \mid t), \kappa_{f}\left(x^{*}(N \mid t), \bar{x}^{*}(t)\right)\right) \\
& \quad+V_{f}\left(f\left(x^{*}(N \mid t), \kappa_{f}\left(x^{*}(N \mid t), \bar{x}^{*}(t)\right)\right), \bar{x}^{*}(t)\right)-V_{f}\left(x^{*}(N \mid t), \bar{x}^{*}(t)\right)+\gamma(t) \ell\left(\bar{x}^{*}(t), \bar{u}^{*}(t)\right) \\
& \stackrel{\text { Ass.1(iii) }}{\leq}-\ell(x(t), u(t))+(1+\gamma(t)) \ell\left(\bar{x}^{*}(t), \bar{u}^{*}(t)\right) .
\end{aligned}
$$

From here, the remainder of the proof follows the lines of the proof of Theorem 1 in [16].

Given that $\kappa_{\infty}$ is an upper bound on the closed-loop asymptotic average performance, it is of interest to determine the value of $\kappa_{\infty}$. In [16], the following rather conceptual results were obtained. Let $\omega_{B}\left(x_{0}\right)$ be the $\omega$-limit set of the closed-loop state sequence (7) starting at $x_{0}$, i.e., $\omega_{B}\left(x_{0}\right):=\left\{y \in \mathbb{Z}_{\mathbb{X}}: \exists t_{n} \rightarrow+\infty\right.$ s.t $x(0)=x_{0}$ and $\left.\lim _{n \rightarrow \infty} x\left(t_{n}\right)=y\right\}$, where $x(\cdot)$ is the closed-loop solution arising from (7).

Theorem 2 ([16, Theorem 2]). (i) Suppose that Assumption 1 is satisfied and the update rule B is such that for all sequences $x(\cdot)$ and $\kappa(\cdot)$, regarded as open-loop input signals in (8), it holds that

$$
\kappa_{\infty}-\liminf _{t \rightarrow \infty} \ell_{\min }(x(t))>0 \Rightarrow \liminf _{t \rightarrow \infty} \beta(t)=\infty
$$

Then, for the closed-loop system (7) and (8), it holds that $\lim _{t \rightarrow \infty} \ell_{\min }(x(t))$ exists and

$$
\kappa_{\infty}=\lim _{t \rightarrow \infty} \ell_{\min }(x(t)) \leq \inf _{y \in \omega_{B}\left(x_{0}\right)} \bar{\ell}_{\min }(y)
$$


(ii) Suppose that Assumption 1 is satisfied and the update rule $B$ is such that for all sequences $x(\cdot)$ and $\kappa(\cdot)$, regarded as open-loop input signals in (8), it holds that

$$
\kappa_{\infty}-\limsup _{t \rightarrow \infty} \ell_{\min }(x(t))>0 \Rightarrow \limsup _{t \rightarrow \infty} \beta(t)=\infty,
$$

Then, for the closed-loop system (7) and (8), it holds that

$$
\kappa_{\infty}=\limsup _{t \rightarrow \infty} \ell_{\min }(x(t)) \leq \sup _{y \in \omega_{B}\left(x_{0}\right)} \bar{\ell}_{\min }(y)
$$

Proof: Modulo some minor modifications, Theorem 2 can be proven as [16, Theorem 2], which considered the generalized terminal equality constraint setting ${ }^{4}(3)-(4)$.

In [16], we proposed several specific update rules $B$ fulfilling the conditions of Theorems 1 and 2 . The interested reader is referred to [16, Sections 4-5] for a detailed description and discussion of these update rules.

\section{Improved a priori bounds for economic MPC with self-tuning terminal cost}

Loosely speaking, Theorem 2 means that $\kappa_{\infty}$ is equal to the best robustly achievable steady-state cost from the $\omega$-limit set of solutions resulting in closed-loop. While this is a desirable behavior, the result is of rather conceptual nature, in the sense that no a priori verifiable bounds for $\kappa_{\infty}$ are given. As a main result of this paper, we now show that when using the generalized terminal region constraint as in (5)-(6), $\kappa_{\infty}$ is a local minimum of the stage cost on the feasible steady-state set, i.e., to the following optimization problem:

$$
\min _{(x, u) \in \overline{\mathbb{Z}}, x=f(x, u)} \ell(x, u) .
$$

Let $\Omega_{\infty}:=\left\{(\bar{x}, \bar{u}) \in \overline{\mathbb{Z}}: \exists t_{n} \rightarrow+\infty\right.$ s.t $\left.\lim _{n \rightarrow \infty}\left(\bar{x}^{*}\left(t_{n}\right), \bar{u}^{*}\left(t_{n}\right)\right)=(\bar{x}, \bar{u})\right\}$, where $\left(\bar{x}^{*}\left(t_{n}\right), \bar{u}^{*}\left(t_{n}\right)\right)$ comes from the optimal solution to problem (5)-(6) at time $t_{n}$. By definition of $\kappa_{\infty}$, we have $\ell(\bar{x}, \bar{u})=\kappa_{\infty}$ for all $(\bar{x}, \bar{u}) \in \Omega_{\infty}$. Note that while $\ell\left(\bar{x}^{*}(t), \bar{u}^{*}(t)\right)$ converges to $\kappa_{\infty}$ for $t \rightarrow \infty$ as discussed above, $\left(\bar{x}^{*}(t), \bar{u}^{*}(t)\right)$ does not necessarily converge and hence $\Omega_{\infty}$ is not necessarily a singleton.

\footnotetext{
${ }^{4}$ In particular, one step in the proof of [16, Theorem 2] relies on [15, Proposition 2], which can straightforwardly be extended to the setting considered here, i.e., with a generalized terminal region constraint as in (5)-(6).
} 
Theorem 3. (i) Suppose that the conditions of Theorem 2(i) are satisfied. Then each $(\bar{x}, \bar{u}) \in \Omega_{\infty}$ is a local minimizer of problem (20).

(ii) Suppose that the conditions of Theorem 2(ii) are satisfied. Then some $(\bar{x}, \bar{u}) \in \Omega_{\infty}$ is a local minimizer of problem (20).

We obtain the following corollary from Theorem 3.

Corollary 1. Suppose that the conditions of Theorem 3(i) or (ii) are satisfied. Then $\kappa_{\infty}$ is a local minimum of problem (20).

Proof: The result immediately follows from Theorem 3 by noting that $\ell(\bar{x}, \bar{u})=\kappa_{\infty}$ for all $(\bar{x}, \bar{u}) \in$ $\Omega_{\infty}$ and the fact that in both cases, at least one steady-state $(\bar{x}, \bar{u}) \in \Omega_{\infty}$ is a local minimizer of problem (20).

Remark 3. We note that the results of Theorem 3 and Corollary 1 can in general not be established for the generalized terminal equality constraint setting (3)-(4), but only for the generalized terminal region setting (5)-(6). A simple illustration of this fact is given in Example 2 in Section 5.

In order to prove Theorem 3, we need the following auxiliary result.

Lemma 1. Let $c:=\left(1-\frac{\lambda_{\min }(Q)}{\lambda_{\max }(P)}\right) / \lambda_{\min }(P)$. For each steady-state $(\bar{x}, \bar{u}) \in \overline{\mathbb{Z}}$ and each $x \in \mathbb{X}^{f}(\bar{x})$, it holds that $x^{+}=f\left(x, \kappa_{f}(x, \bar{x})\right) \in \mathbb{X}^{f}(y)$, for all $y \in B_{\varepsilon}(\bar{x})$ with

$$
\varepsilon:=\left(-\sqrt{c}+\sqrt{c+\frac{\lambda_{\min }(Q)}{\lambda_{\max }(P)^{2}}}\right) \sqrt{\alpha} .
$$

Proof: Due to Assumption 1(ii), for each steady-state $(\bar{x}, \bar{u})$ and each $x \in \mathbb{X}^{f}(\bar{x})$ we have

$$
\begin{aligned}
E\left(x^{+}, \bar{x}\right) & \leq E(x, \bar{x})-(x-\bar{x})^{T} Q(x-\bar{x}) \leq E(x, \bar{x})-\lambda_{\min }(Q)|x-\bar{x}|^{2} \\
& \leq E(x, \bar{x})-\frac{\lambda_{\min }(Q)}{\lambda_{\max }(P)} E(x, \bar{x}) \leq\left(1-\frac{\lambda_{\min }(Q)}{\lambda_{\max }(P)}\right) \alpha .
\end{aligned}
$$

This means that $x^{+}$lies in the tightened set $\widetilde{\mathbb{X}}^{f}(\bar{x}):=\left\{x \in \mathbb{R}^{n}: E(x, \bar{x}) \leq c \lambda_{\min }(P) \alpha\right\} \subset \mathbb{X}^{f}(\bar{x})$. We now want to find the maximum $\varepsilon>0$ such that $\widetilde{\mathbb{X}}^{f}(\bar{x}) \subseteq \mathbb{X}^{f}(y)$ for all $y \in B_{\varepsilon}(\bar{x})$ (see an exemplary illustration in Figure 1). This is the case if $E(x, y) \leq \alpha$ for all $x \in \widetilde{\mathbb{X}}^{f}(\bar{x})$ and all 


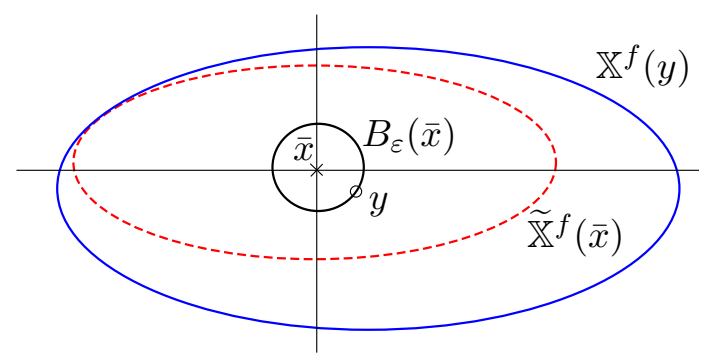

Figure 1: Illustration of the sets $\mathbb{X}^{f}(y), \tilde{\mathbb{X}}^{f}(\bar{x})$ (dashed) and $B_{\varepsilon}(\bar{x})$, as well as $y$ ("o") and $\bar{x}$ ("x").

$y \in B_{\varepsilon}(\bar{x})$. We obtain

$$
\begin{aligned}
(x-y)^{T} P(x-y) & =(x-\bar{x}+\bar{x}-y)^{T} P(x-\bar{x}+\bar{x}-y) \\
& =(x-\bar{x})^{T} P(x-\bar{x})+2(x-\bar{x})^{T} P(\bar{x}-y)+(\bar{x}-y)^{T} P(\bar{x}-y) \\
& \leq c \lambda_{\min }(P) \alpha+2 \sqrt{c \alpha} \lambda_{\max }(P) \varepsilon+\lambda_{\max }(P) \varepsilon^{2}
\end{aligned}
$$

where the last inequality comes from the fact that $(x-\bar{x})^{T} P(x-\bar{x}) \leq c \lambda_{\min }(P) \alpha$ and $|x-\bar{x}| \leq \sqrt{c \alpha}$ for all $x \in \widetilde{\mathbb{X}}^{f}(\bar{x})$, and $|\bar{x}-y| \leq \varepsilon$ for all $y \in B_{\varepsilon}(\bar{x})$. The right hand side of (23) is less or equal than $\alpha$ if $\varepsilon$ satisfies the bound (21), which follows from solving the corresponding quadratic equation. This concludes the proof of Lemma 1.

Proof of Theorem 3: (i) Assume for contradiction that there exists $(\hat{x}, \hat{u}) \in \Omega_{\infty}$ which is not a local minimizer of problem (20). This means that for each $\delta>0$, there exists another steady-state $\left(x^{\prime}, u^{\prime}\right) \in B_{\delta}(\hat{x}, \hat{u}) \cap \overline{\mathbb{Z}}$ with $\ell\left(x^{\prime}, u^{\prime}\right)<\ell(\hat{x}, \hat{u})$. Fix some $\delta \leq \varepsilon / 2$, with $\varepsilon$ as in (21). By definition of $\Omega_{\infty}$, there exists an infinite sequence of time instants $\left\{t_{i}\right\}$ such that $\left(\bar{x}^{*}\left(t_{i}\right), \bar{u}^{*}\left(t_{i}\right)\right) \in B_{\delta}(\hat{x}, \hat{u}) \cap \overline{\mathbb{Z}}$. Then, at the respective next time instant $t_{i}+1$, a feasible solution to problem (5)-(6) is given by the end piece of the previously optimal solution appended by the terminal controller, i.e., $\tilde{\mathbf{u}}\left(t_{i}+1\right):=$ $\left\{u^{*}\left(1 \mid t_{i}\right), \ldots, \kappa_{f}\left(x^{*}\left(N \mid t_{i}\right), \bar{x}^{*}\left(t_{i}\right)\right)\right\}$, and $\left(\bar{x}\left(t_{i}+1\right), \bar{u}\left(t_{i}+1\right)\right):=\left(\bar{x}^{*}\left(t_{i}\right), \bar{u}^{*}\left(t_{i}\right)\right)$. The corresponding feasible predicted state sequence is $\tilde{\mathbf{x}}\left(t_{i}+1\right):=\left\{x^{*}\left(1 \mid t_{i}\right), \ldots, f\left(x^{*}\left(N \mid t_{i}\right), \kappa_{f}\left(x^{*}\left(N \mid t_{i}\right), \bar{x}^{*}\left(t_{i}\right)\right)\right)\right\}$. By Lemma 1, it follows that $f\left(x^{*}\left(N \mid t_{i}\right), \kappa_{f}\left(x^{*}\left(N \mid t_{i}\right), \bar{x}^{*}\left(t_{i}\right)\right)\right) \in \mathbb{X}^{f}(y)$, for all $y \in B_{\varepsilon}\left(\bar{x}^{*}\left(t_{i}\right)\right)$ with $\varepsilon$ given by (21). As $\delta \leq \varepsilon / 2$ and hence $x^{\prime} \in B_{\varepsilon}\left(\bar{x}^{*}\left(t_{i}\right)\right)$, it follows that $f\left(x^{*}\left(N \mid t_{i}\right), \kappa_{f}\left(x^{*}\left(N \mid t_{i}\right), \bar{x}^{*}\left(t_{i}\right)\right)\right) \in$ $\mathbb{X}^{f}\left(x^{\prime}\right)$. But then another feasible solution to problem (5)-(6) at each time $t_{i}+1$ is given by $\tilde{\mathbf{u}}\left(t_{i}+1\right)$ and $\left(\bar{x}\left(t_{i}+1\right), \bar{u}\left(t_{i}+1\right)\right):=\left(x^{\prime}, u^{\prime}\right)$. Thus, by definition of $\ell_{\min }$ in $(10)$, we obtain $\lim _{i \rightarrow \infty} \ell_{\min }\left(x\left(t_{i}\right)\right) \leq \ell\left(x^{\prime}, u^{\prime}\right)<\ell(\hat{x}, \hat{u})=\kappa_{\infty}$. This is a contradiction to the fact that $\kappa_{\infty}=$ $\lim _{t \rightarrow \infty} \ell_{\min }(x(t))$ as shown in Theorem 2(i). 
(ii) Assume for contradiction that each $(\bar{x}, \bar{u}) \in \Omega_{\infty}$ is not a local minimizer of problem (20). This means that for each $\delta>0$ and each $(\bar{x}, \bar{u}) \in \Omega_{\infty}$, there exists another steady-state $\left(x^{\prime}, u^{\prime}\right) \in$ $B_{\delta}(\bar{x}, \bar{u}) \cap \overline{\mathbb{Z}}$ and (due to compactness of $\Omega_{\infty}$ ) a constant $\ell^{\prime}$ such that $\ell\left(x^{\prime}, u^{\prime}\right) \leq \ell^{\prime}<\ell(\bar{x}, \bar{u})$. Fix some $\delta \leq \varepsilon / 2$, with $\varepsilon$ as in (21). By definition of $\Omega_{\infty}$, there exists $\bar{t} \in \mathbb{I}_{\geq 0}$ such that $\left(\bar{x}^{*}(t), \bar{u}^{*}(t)\right) \in \cup_{(\bar{x}, \bar{u}) \in \Omega_{\infty}} B_{\delta}(\bar{x}, \bar{u}) \cap \overline{\mathbb{Z}}$ for all $t \geq \bar{t}$. Using the same argument as above in the proof of Theorem 3(i), i.e., by evoking Lemma 1 , if follows that $\ell_{\min }(x(t)) \leq \ell^{\prime}<\kappa_{\infty}$ for all $t \geq \bar{t}$, and hence in particular also $\lim \sup _{t \rightarrow \infty} \ell_{\min }(x(t)) \leq \ell^{\prime}<\kappa_{\infty}$. But this is a contradiction to the fact that $\kappa_{\infty}=\lim \sup _{t \rightarrow \infty} \ell_{\min }(x(t))$ as shown in Theorem 2(ii).

For the case of linear systems with convex cost function and constraint set $\overline{\mathbb{Z}}$, we have the following corollary of Theorem 3 (respectively, of Corollary 1).

Corollary 2. Suppose that the conditions of Theorem 2(i) or (ii) are satisfied, that the system (1) is linear, and that the stage cost $\ell$ and the constraint set $\overline{\mathbb{Z}}$ are convex. Then $\kappa_{\infty}$ is the global minimum of problem (20).

Remark 4. Corollary 2 combined with Theorem 1 means that the asymptotic average performance is at least as good as the cost of the optimal steady-state in $\overline{\mathbb{Z}}$. This recovers the result obtained in [2] in a setting where the optimal steady-state was used as a fixed terminal state constraint. Namely, in [2] it was shown that the asymptotic average performance is at least as good as the cost of the optimal steady-state in $\mathbb{Z}$, i.e., $\ell\left(x_{s}, u_{s}\right)$ according to (2). In Section 4 (see Remark 5) we comment on how the generalized terminal region can be chosen such that $\overline{\mathbb{Z}}$ can be any compact set $\overline{\mathbb{Z}} \subseteq \operatorname{int}(\mathbb{Z})$.

\section{Calculation of terminal ingredients}

We discuss next how the terminal ingredients can be calculated such that Assumption 1 is satisfied. The construction builds on the results in [3], where a terminal region around a fixed steady-state is constructed in case that both $f$ and $\ell$ are twice continuously differentiable, which we also assume in the following. We first consider the case of linear systems, where a general design procedure can be derived. After that, we discuss possible extensions to nonlinear systems; however, the resulting conditions in this case are conservative and hence might only be applicable to special classes of nonlinear systems. 


\subsection{Linear systems}

Suppose that the system map $f$ in (1) is linear, i.e., $f(x, u)=A x+B u$, with $(A, B)$ stabilizable. Choose any controller such that $A_{K}:=A+B K$ is stable. Next, for a given $Q>0$, one can compute the matrix $P$ as the solution to the Lyapunov equation $A_{K}^{T} P A_{K}-P=-Q$. Then, with $\kappa_{f}(x, \bar{x}):=K(x-\bar{x})+\bar{u}$, requirement (ii) of Assumption 1 is satisfied for each $\bar{x}$. Now define $\bar{\ell}(x):=\ell(x, K(x-\bar{x})+\bar{u})-\ell(\bar{x}, \bar{u})$. By a straightforward modification of [3, Lemma 22], it follows that there exists some symmetric matrix $H$ such that $H-\bar{\ell}_{x x}(x) \geq 0$ for all $x \in \mathbb{Z}_{\mathbb{X}}$ and $(\bar{x}, \bar{u}) \in \mathbb{Z}$. Following the same procedure as in [3, Section 4.1], one can show that requirement (iii) of Assumption 1 is satisfied for all $(\bar{x}, \bar{u}) \in \mathbb{Z}$ and all $x \in \mathbb{X}^{f}(\bar{x})$ if the terminal cost $V_{f}$ is defined as

$$
V_{f}(x, \bar{x})=(1 / 2)(x-\bar{x})^{T} G(x-\bar{x})+\left(\bar{\ell}_{x}(\bar{x})\right)^{T}\left(I-A_{K}\right)^{-1}(x-\bar{x}),
$$

where $G$ is the solution to the Lyapunov equation $A_{K}^{T} G A_{K}-G=-H$.

Finally, it remains to determine the set $\overline{\mathbb{Z}}$ such that condition $(i)$ in Assumption 1 can be satisfied for all steady-states $(\bar{x}, \bar{u}) \in \overline{\mathbb{Z}}$, i.e., such that state and input constraints are satisfied in the terminal region around each steady-state $(\bar{x}, \bar{u}) \in \overline{\mathbb{Z}}$. Let $\mathbb{U}^{f}(\bar{x}):=\left\{\kappa_{f}(x, \bar{x}): x \in \mathbb{X}^{f}(\bar{x})\right\}$. Due to the definition of the terminal region $\mathbb{X}^{f}$ and the terminal controller $\kappa_{f}$, we have that $\mathbb{X}^{f}(\bar{x})=\{\bar{x}\} \oplus \mathbb{X}^{f}(0)$ and $\mathbb{U}^{f}(\bar{x})=\{\bar{u}\} \oplus K \mathbb{X}^{f}(0)$. Hence condition $(i)$ in Assumption 1 is satisfied if the set $\overline{\mathbb{Z}}$ is defined as

$$
\overline{\mathbb{Z}}=\mathbb{Z} \ominus\left(\mathbb{X}^{f}(0) \times K \mathbb{X}^{f}(0)\right)
$$

Remark 5. Some comments on the terminal regions $\mathbb{X}^{f}(\bar{x})$ and the set $\overline{\mathbb{Z}}$ are in order. Recalling the definition of $\mathbb{X}^{f}(\bar{x}):=\left\{x \in \mathbb{R}^{n}: E(x, \bar{x}) \leq \alpha\right\}$ from Assumption 1, one can see that for $\alpha \rightarrow 0$, it holds that $\overline{\mathbb{Z}}$ approaches $\mathbb{Z}$. Hence in order to obtain the strongest results in Theorem 3, it would be desirable to choose $\alpha$ small. Also, one can see that for $\alpha \rightarrow 0$, the feasible set of problem (5)-(6) approaches the feasible set of problem (3)-(4). On the other hand, larger values of $\alpha$, i.e., larger terminal sets, might result in a larger feasible set of problem (5)-(6). In fact, instead of using a constant $\alpha$ for the definition of the terminal regions $\mathbb{X}^{f}(\bar{x})$, one can also define $\alpha$ as a function of $\bar{x}$ given by

$$
\alpha(\bar{x}):=\max _{\left(x, \kappa_{f}(x, \bar{x})\right) \in \mathbb{Z} \text { for all } x \in \mathbb{X}^{f}(\bar{x})} \alpha,
$$


in which case $\cup_{\bar{x} \in \mathbb{Z}_{\mathbb{X}}} \mathbb{X}^{f}(\bar{x})$ can be seen as an ellipsoidal approximation of the maximal invariant set for tracking (projected on $\mathbb{X}$ ) as used in [12, 13]. Then, condition (i) in Assumption 1 is satisfied for $\overline{\mathbb{Z}}=\mathbb{Z}$ by definition of $\alpha(\bar{x})$. Furthermore, as $\alpha(\bar{x})$ is continuous in $\bar{x}$ according to (26) (as $\kappa_{f}$ is continuous), for any compact set $\overline{\mathbb{Z}} \subseteq \operatorname{int}(\mathbb{Z})$ one can show similarly to the proof of Lemma 1 that there exists an $\varepsilon>0$ such that $\widetilde{\mathbb{X}}^{f}(\bar{x}) \subseteq \mathbb{X}^{f}(y)$ for all $y \in B_{\varepsilon}(\bar{x})$ and all $\bar{x} \in \overline{\mathbb{Z}}$. Hence Theorem 3 is valid with $\overline{\mathbb{Z}}$ in (20) being any compact set $\overline{\mathbb{Z}} \subseteq \operatorname{int}(\mathbb{Z})$. However, we note that (analytically) calculating $\alpha(\bar{x})$ might in general be difficult (depending on the shape of $\mathbb{Z}$ and $\kappa_{f}$ ). In this case, another relaxation compared to a fixed $\alpha$ can be used. Namely, given some initial value $\alpha(0)=\alpha_{0}>0$ and some $0<\alpha_{\min } \leq \alpha_{0}$, one can, at subsequent time instants, define

$$
\alpha(t+1):=\max \left\{\left(1-(1-\theta) \frac{\lambda_{\min }(Q)}{\lambda_{\max }(P)}\right) \alpha(t), \alpha_{\min }\right\}
$$

for some $0<\theta<1$ and in (6d) use the time-varying terminal regions $\mathbb{X}^{f}(\bar{x}, t):=\left\{x \in \mathbb{R}^{n}\right.$ : $E(x, \bar{x}) \leq \alpha(t)\}$. Then, condition $(i)$ in Assumption 1 is satisfied for $\overline{\mathbb{Z}}=\overline{\mathbb{Z}}(t):=\mathbb{Z} \ominus\left(\mathbb{X}^{f}(0, t) \times\right.$ $\left.K \mathbb{X}^{f}(0, t)\right)$. Furthermore, analogous to Lemma 1 , one can show that for each $t \in \mathbb{I}_{\geq 0}, \widetilde{\mathbb{X}}^{f}(\bar{x}, t) \subseteq$ $\mathbb{X}^{f}(y, t+1)$ for all $y \in B_{\varepsilon}(\bar{x})$ with $\varepsilon:=\left(-\sqrt{c}+\sqrt{c+\theta \frac{\lambda_{\min }(Q)}{\lambda_{\max }(P)^{2}}}\right) \sqrt{\alpha(t)}$. Thus, as $\alpha_{\min }$ can be chosen arbitrarily small and for each $\alpha_{\min }>0$ there exists some $\hat{t}$ such that $\alpha(t)=\alpha_{\min }$ for all $t \in \mathbb{I}_{\geq \hat{t}}$, one can again apply Theorem 3 with $\overline{\mathbb{Z}}$ in (20) being any compact set $\overline{\mathbb{Z}} \subseteq \operatorname{int}(\mathbb{Z})$. Finally, we note that the two methods described above for using a non-constant $\alpha$ only result in very little additional online computational effort. Namely, the state-dependent $\alpha(\bar{x})$ as given in (26) would have to be calculated offline, and defining $\alpha(t)$ as in $(27)$ is a simple algebraic operation.

\subsection{Nonlinear systems}

We now present some extensions of the results of Section 4.1 to show how the terminal ingredients can be calculated such that Assumption 1 is satisfied for nonlinear systems. The resulting conditions are, however, merely sufficient and hence in general conservative. Denote the linearization of system (1) at some steady-state $\bar{z}:=(\bar{x}, \bar{u})$ by $A(\bar{z}):=(\partial f / \partial x)(\bar{x}, \bar{u})$ and $B(\bar{z}):=(\partial f / \partial u)(\bar{x}, \bar{u})$. As $\mathbb{Z}$ is compact and $A(\bar{z})$ and $B(\bar{z})$ are continuous in $\bar{z}$ (as $f, \ell \in C^{2}$ ), $(A(\bar{z}), B(\bar{z})$ ) is contained in some compact set $\overline{\mathcal{A}}$ for all steady-states $\bar{z} \in \mathbb{Z}$. Denote by $\mathcal{A}$ a convex polytopic outer approximation of $\overline{\mathcal{A}}$. In order to satisfy conditions (ii) and (iii) of Assumption 1, we require that the matrices $P$ (defining the terminal regions $\mathbb{X}^{f}(\bar{x})$ ) and $K$ (the terminal controller gain) satisfy the following inequality for all $(A(\bar{z}), B(\bar{z})) \in \overline{\mathcal{A}}$ and $Q:=H+\delta I$ with $H$ as defined in Section 4.1 and 
$\delta>0$ such that $Q>0$ :

$$
(A(\bar{z})+B(\bar{z}) K)^{T} P(A(\bar{z})+B(\bar{z}) K)-P \leq-Q
$$

For a fixed $\bar{z}$, this inequality can be transformed into an equivalent linear matrix inequality (LMI) which is affine in $(A(\bar{z}), B(\bar{z}))$ by using the Schur complement (see, e.g., [18]). Then, in order to be satisfied for all $(A(\bar{z}), B(\bar{z})) \in \overline{\mathcal{A}}$, it is sufficient that these LMIs are satisfied for all extreme points of the polytope $\mathcal{A}$, as $\mathcal{A}$ is convex and the LMIs are affine in $(A(\bar{z}), B(\bar{z}))$. Hence the requirement that $(28)$ has to hold for all $A(\bar{z}), B(\bar{z}) \in \overline{\mathcal{A}}$ can be translated into a finite number of LMIs to be satisfied. Now if (28) holds for all $A(\bar{z}), B(\bar{z}) \in \overline{\mathcal{A}}$, then one can show analogous to [3, Section 4.1] that there exists a constant $\alpha_{\max }>0$ such that both conditions (ii) and (iii) of Assumption 1 are satisfied for all $\alpha \leq \alpha_{\max }$ and terminal cost given by (24) (with $A_{K}:=A(\bar{z})+B(\bar{z}) K$ and $G=P)$ and auxiliary terminal controller $\kappa_{f}(x, \bar{x}):=K(x-\bar{x})+\bar{u}$. Furthermore, as in Section 4.1, condition $(i)$ in Assumption 1 is satisfied if the set $\overline{\mathbb{Z}}$ is defined by (25).

Remark 6. The considerations of Remark 5 also apply to nonlinear systems, i.e., state- or timedependent values of $\alpha$ are possible. However, one has to restrict $\alpha$ to be less or equal to $\alpha_{\max }$.

Remark 7. As one can see from the definition in (24), the terminal cost function $V_{f}$ contains the term $\left(I-A_{K}\right)^{-1}$. For linear systems, $A_{K}$ is a fixed matrix and hence the matrix inversion can be done offline. On the other hand, when considering nonlinear systems, $A_{K}$ is given by $A_{K}:=A(\bar{x})+B(\bar{x}) K$, and hence analytically computing the inverse of $\left(I-A_{K}\right)$ as a function of $\bar{x}$ might in general not be possible. In this case, the respective matrix inversions have to be done online when evaluating $V_{f}$ for a given $\bar{x}$; this, however, might result in a computationally high burden when solving the optimization problem (5)-(6).

Remark 8. Note that the above derived conditions are conservative since we require the matrices $P$ and $K$ to be constant, i.e., inequality (28) has to be satisfied for all $(A(\bar{z}), B(\bar{z})) \in \overline{\mathcal{A}}$ with constant $P$ and $K$, which might in general be possible for "mild" nonlinearities only. A possible relaxation of this would be to allow $P$ and $K$ to depend on $\bar{z}$. Similar to what was discussed in Remark 5 for the case where $\alpha$ depends on $\bar{x}$, one can show that if $P(\bar{z})$ is continuous in $\bar{z}$, then again Lemma 1 can be appropriately modified and hence Theorem 3 is still valid. Note that a continuous $P(\bar{z})$ 
can in principle be computed as outlined in the following, if $(A(\bar{z}), B(\bar{z}))$ is stabilizable for all $\bar{z} \in \overline{\mathbb{Z}}$. Namely, as discussed above, both $A(\bar{z})$ and $B(\bar{z})$ are continuous in $\bar{z}$. One can then use the pole-placement explicit formula with a fixed desired closed-loop characteristic polynomial to obtain a continuous closed-loop matrix $A(\bar{z})+B(\bar{z}) K(\bar{z})$ (at least, this can be done for single-input systems). Then, solving the Lyapunov equation (28) yields a matrix $P(\bar{z})$ which is continuous in $\bar{z}$. However, we note that this procedure in general cannot be translated into a fixed number of inequalities to be solved (as was the case above for fixed $P$ and $K$ ) and computing $K(\bar{z})$ and $P(\bar{z})$ analytically as a function of $\bar{z}$ is in general not possible, which means that finding non-constant continuous $K(\bar{z})$ and $P(\bar{z})$ might not be tractable.

\section{Numerical Examples}

Example 1: Consider system $(1)$ with $f(x, u)=1.1 x+u$ and $\mathbb{Z}=[-5,5] \times[-1,1]$. The stage cost function $\ell$ is given by $\ell(x, u)=(1 / 20)(x+4)(x+2)(x-2)(0.9 x-4)-u^{2}$. The terminal cost $V_{f}$, the auxiliary terminal controllers $\kappa_{f}(x, \bar{x})$, the terminal regions $\mathbb{X}^{f}(\bar{x})=\left\{x:(x-\bar{x})^{2} \leq 0.25\right\}$ and the set $\overline{\mathbb{Z}}$ (according to (25)) were calculated such that Assumption 1 is satisfied. The set of all steady-states in $\overline{\mathbb{Z}}$ is $\bar{S}:=\left\{(\bar{x}, \bar{u}): \bar{u}=-0.1 \bar{x}, \bar{x} \in\left[\begin{array}{ll}-4.5 & 4.5\end{array}\right]\right\}$. Figure 2(a) shows the stage cost function $\ell$ restricted to the steady-state set, i.e., for $u=-0.1 x$. One can see that problem (20) has a local minimum for $x \approx-3.175$ with $\ell(x,-0.1 x) \approx-1.82$ and a global minimum for $x \approx 3.47$, i.e., $\left(x_{s}, u_{s}\right) \approx(3.47,0.347)$ with $\ell\left(x_{s}, u_{s}\right) \approx-2.75$. As discussed in the Introduction, the major advantage of using a generalized terminal constraint instead of a fixed terminal constraint is that a possibly much larger region of attraction is obtained. Namely, even for prediction horizon $N=1$, all initial conditions $x_{0} \in \mathbb{Z}_{\mathbb{X}}=[-5,5]$ are feasible for problem (5)-(6) (under suitable initialization of $\kappa$, compare [16, Section 5]). On the other hand, when using a fixed terminal region around the optimal steady-state $x_{s}$ (with the same size as above, i.e., $\alpha=0.25$ ), only initial conditions $x_{0} \in[1.79,4.52]$ are feasible for $N=1$, and a prediction horizon of $N=10$ is needed such that again all initial conditions $x_{0} \in \mathbb{Z}_{\mathbb{X}}=[-5,5]$ are feasible. Figures 2(b)-2(d) show closed-loop sequences of $\kappa, x$ and $u$ with two different initial conditions $x_{0}$ and prediction horizon $N=4$, using the generalized terminal region setting (5)-(6). The update rule (8) for the terminal weight $\beta$ was chosen such that both Assumption 2 and (16) are satisfied (see [16, Section 4.2] for different such update rules). As guaranteed by Theorem 3 , one can see that $\kappa$ converges to one of the two minima 


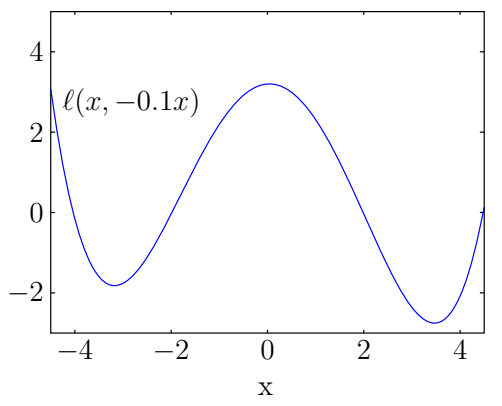

(a) Steady-state cost.

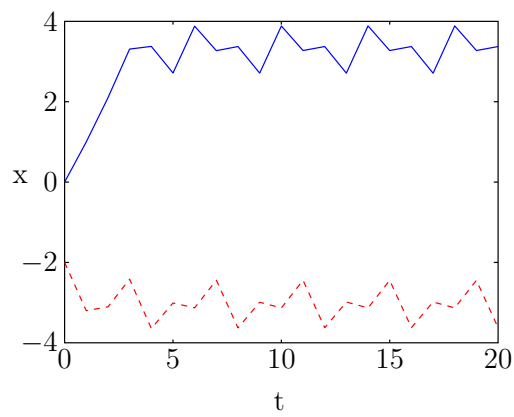

(c) Closed-loop state sequences.

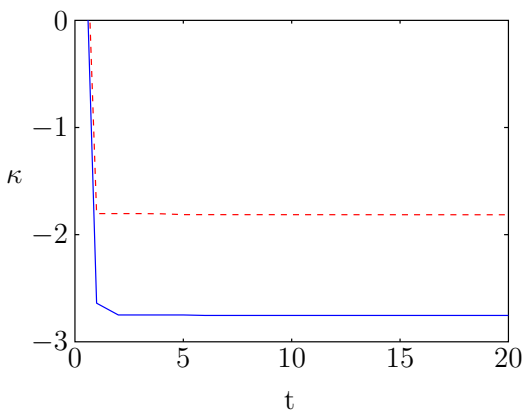

(b) Closed-loop $\kappa$ sequences.

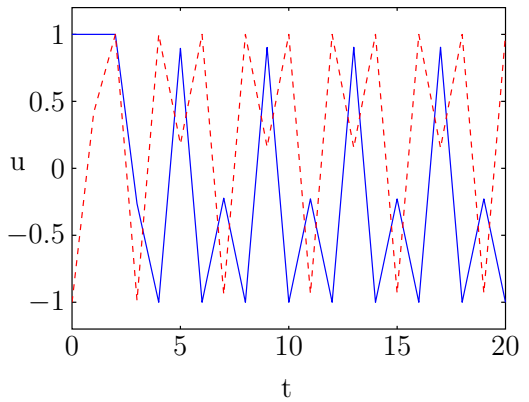

(d) Closed-loop input sequences.

Figure 2: Steady-state cost (a) and closed-loop sequences (b)-(d) in Example 1 with initial conditions $x_{0}=0$ (solid) and $x_{0}=-2$ (dashed).

of problem (20), depending on the initial condition. The closed-loop input and state sequences are non-convergent, resulting in a better average performance than steady-state operation. As guaranteed by Theorem 1, we obtain $\lim \sup _{T \rightarrow \infty}(1 / T) \sum_{t=0}^{T-1} \ell(x(t), u(t))=-2.11 \leq-1.82=$ $\kappa_{\infty}$ for $x_{0}=-2$ and $\lim \sup _{T \rightarrow \infty}(1 / T) \sum_{t=0}^{T-1} \ell(x(t), u(t))=-2.91 \leq-2.75=\kappa_{\infty}$ for $x_{0}=0$. Furthermore, as discussed above, for long enough prediction horizons $(N \geq 10)$ the optimal steadystate $x_{s}$ (respectively, the terminal region around it) can be reached from all $x \in \mathbb{Z}_{\mathbb{X}}=[-5,5]$. In this case, one observes that as guaranteed by Theorem $2, \kappa$ converges to $\ell\left(x_{s}, u_{s}\right)$ for all initial conditions $x_{0} \in \mathbb{Z}_{\mathbb{X}}=[-5,5]$.

Example 2: The following example illustrates that Theorem 3 can in general only be established when using the generalized terminal region setting (5)-(6), but not for the generalized 
terminal equality constraint setting (3)-(4). Consider the system

$$
\begin{aligned}
& x_{1}(t+1)=x_{1}(t)+x_{2}(t) \cos \left(0.5 x_{1}(t)\right) \\
& x_{2}(t+1)=x_{2}(t)+u(t)
\end{aligned}
$$

with state and input constraint set $\mathbb{Z}=[-2,2]^{3}$ and cost function $\ell(x, u)=x_{1}^{2}$. The set of all steady-states in $\mathbb{Z}$ is given by $S:=\left\{(\bar{x}, \bar{u}): \bar{x}_{1} \in[-2,2], \bar{x}_{2}=\bar{u}=0\right\}$. The terminal cost $V_{f}$, the auxiliary terminal controllers $\kappa_{f}(x, \bar{x})$, the terminal regions $\mathbb{X}^{f}(\bar{x})=\left\{x:(x-\bar{x})^{T} P(x-\bar{x}) \leq\right.$ $0.37\}$ and the set $\overline{\mathbb{Z}}$ were calculated according to the procedure proposed in Section 4.2 such that Assumption 1 is satisfied. Namely, for all $\bar{z} \in S, A(\bar{z})$ is contained in a polytope with extreme points $A_{1}=\left[\begin{array}{ll}1 & 1 \\ 0 & 1\end{array}\right]$ (resulting from $\bar{x}_{1}=0$ ) and $A_{2}=\left[\begin{array}{cc}1 & \cos (1) \\ 0 & 1\end{array}\right]$ (resulting from $\bar{x}_{1}= \pm 2$ ). The matrices $P=\left[\begin{array}{ll}32.3 & 18.7 \\ 18.7 & 28.2\end{array}\right]$ and $K=\left[\begin{array}{ll}-0.81 & -1.62\end{array}\right]$ were then determined such that the LMIs resulting from (28) are satisfied for both $A_{1}$ and $A_{2}$. Now consider a prediction horizon of $N=1$ and compare the generalized terminal region setting (5)-(6) with the generalized terminal equality constraint setting (3)-(4). For $\kappa_{0}$ sufficiently large, the set of feasible initial states is the same for both problems and given by $\mathcal{X}_{1}:=\left\{x \in[-2,2]^{2}: x_{1}+x_{2} \cos \left(0.5 x_{1}\right) \in[-2,2]\right\}$. When imposing the generalized terminal equality constraint (4d), for each $x_{0} \in \mathcal{X}_{1}$ the only feasible solution to problem (3)-(4) at time $t=0$ is given by $u(0 \mid 0)=-x_{20}, u(1 \mid 0)=0$, and then by $u(0 \mid t)=u(1 \mid t)=0$ for all $t \in \mathbb{I}_{\geq 1}$. This means that for each $x_{0} \in \mathcal{X}_{1}$, the closed-loop solution will converge (in one step) to the steady-state $\bar{x}=\left[\begin{array}{ll}x_{10}+x_{20} \cos \left(0.5 x_{10}\right) & 0\end{array}\right]^{T}$ and then stay there, which in particular implies that $\lim _{T \rightarrow \infty}(1 / T) \sum_{t=0}^{T-1} \ell(x(t), u(t))=\kappa_{\infty}=\left(x_{10}+x_{20} \cos \left(0.5 x_{10}\right)\right)^{2}$. Hence $\kappa_{\infty}$ is not a local minimum of problem (20) (except for the special initial conditions such that $x_{10}+x_{20} \cos \left(0.5 x_{10}\right)=0$ ), i.e., Theorem 3 (and Corollary 1 ) do not hold. On the other hand, when using the generalized terminal region setting (5)-(6), $\kappa$ will converge to 0 for all initial conditions $x_{0} \in \mathcal{X}_{1}$ (see Figure 3). This means that $\kappa_{\infty}$ is the (only) local (and hence global) minimum of problem (20), as guaranteed by Theorem 3 and Corollary 1.

Remark 9. Note that in the present example, the fact that the results of Theorem 3 do not hold for the generalized terminal equality constraint setting (3)-(4) does not depend on the nonlinearity of the system, but is due to the fact that the generalized terminal equality constraint (4d) is (for the 


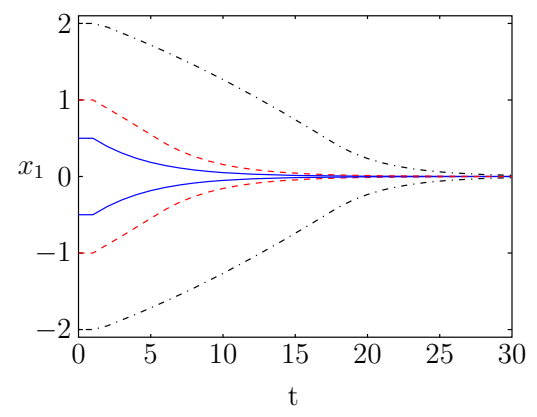

(a) Closed-loop $x_{1}$ sequences.

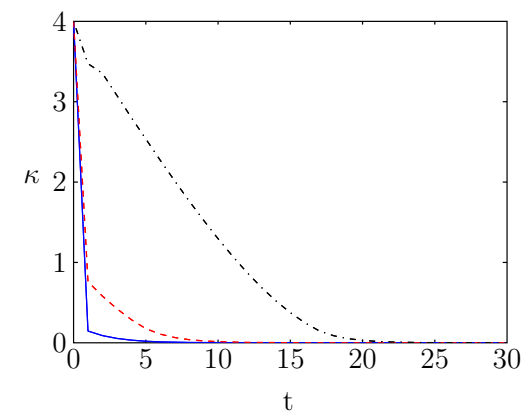

(b) Closed-loop $\kappa$ sequences.

Figure 3: Closed-loop sequences in Example 2 with initial conditions $\kappa_{0}=4$ and $x_{0}=\left[\begin{array}{ll} \pm 2 & 0\end{array}\right]^{T}$ (dash-dotted), $x_{0}=\left[\begin{array}{ll} \pm 1 & 0\end{array}\right]^{T}$ (dashed) and $x_{0}=\left[\begin{array}{ll} \pm 0.5 & 0\end{array}\right]^{T}$ (solid).

given short prediction horizon) much stricter than the generalized terminal region constraint (6d). In fact, the same considerations as above also apply if the term $\cos \left(0.5 x_{1}\right)$ in (29) is, e.g., replaced by 1 and hence the system is linear.

\section{Conclusions}

In this paper, we proposed an economic model predictive control scheme with self-tuning terminal cost and a generalized terminal region constraint. The main advantage of this setting is that improved and a priori verifiable bounds on the closed-loop average performance can be obtained, which is not the case when using a generalized terminal equality constraint. Namely, we showed that the closed-loop average performance is at least as good as a value corresponding to a local minimum of the stage cost function restricted to the set of feasible steady-states (respectively, a global minimum in case of linear systems and convex cost and constraints). On the other hand, the possible limitation of the proposed method is that the (offline) design of the generalized terminal region is more involved compared to the generalized terminal equality constraint. Namely, while a general design procedure was obtained for linear systems, the resulting conditions for nonlinear systems are conservative and hence might in general only be satisfiable for rather "mild " nonlinearities or special system classes. 


\section{References}

[1] M. A. Müller, D. Angeli, F. Allgöwer, Performance analysis of economic MPC with self-tuning terminal cost, in: Proceedings of the American Control Conference, 2014, to appear.

[2] D. Angeli, R. Amrit, J. B. Rawlings, On average performance and stability of economic model predictive control, IEEE Transactions on Automatic Control 57 (7) (2012) 1615-1626.

[3] R. Amrit, J. B. Rawlings, D. Angeli, Economic optimization using model predictive control with a terminal cost, Annual Reviews in Control 35 (2) (2011) 178-186.

[4] L. Grüne, Economic receding horizon control without terminal constraints, Automatica 49 (3) (2013) 725-734.

[5] R. Huang, E. Harinath, L. T. Biegler, Lyapunov stability of economically oriented NMPC for cyclic processes, Journal of Process Control 21 (4) (2011) 501-509.

[6] M. Heidarinejad, J. Liu, P. D. Christofides, Economic model predictive control of nonlinear process systems using Lyapunov techniques, AIChE Journal 58 (3) (2012) 855-870.

[7] M. A. Müller, D. Angeli, F. Allgöwer, R. Amrit, J. B. Rawlings, Convergence in economic model predictive control with average constraints, Automatica, 2014, provisionally accepted for publication.

[8] T. G. Hovgaard, L. F. S. Larsen, J. B. Jørgensen, Flexible and cost efficient power consumption using economic MPC - a supermarket refrigeration benchmark, in: Proceedings of the 50th IEEE Conference on Decision and Control and European Control Conference, 2011, pp. 848854 .

[9] W.-J. Ma, V. Gupta, Desynchronization of thermally-coupled first-order systems using economic model predictive control, in: Proceedings of the 51st IEEE Conference on Decision and Control, 2012, pp. 278-283.

[10] B. Chu, S. Duncan, A. Papachristodoulou, C. Hepburn, Using economic model predictive control to design sustainable policies for mitigating climate change, in: Proceedings of the 51st IEEE Conference on Decision and Control, 2012, pp. 406-411. 
[11] S. Gros, An economic NMPC formulation for wind turbine control, in: Proceedings of the 52nd IEEE Conference on Decision and Control, 2013, pp. 1001-1006.

[12] D. Limon, I. Alvarado, T. Alamo, E. F. Camacho, MPC for tracking piecewise constant references for constrained linear systems, Automatica 44 (9) (2008) 2382-2387.

[13] A. Ferramosca, D. Limon, I. Alvarado, T. Alamo, E. F. Camacho, MPC for tracking with optimal closed-loop performance, Automatica 45 (8) (2009) 1975-1978.

[14] A. Ferramosca, Model predictive control of systems with changing setpoints, Ph.D. thesis, Universidad de Sevilla (2011).

[15] L. Fagiano, A. R. Teel, Generalized terminal state constraint for model predictive control, Automatica 49 (9) (2013) 2622-2631.

[16] M. A. Müller, D. Angeli, F. Allgöwer, Economic model predictive control with self-tuning terminal cost, European Journal of Control 19 (5) (2013) 408-416.

[17] J. B. Rawlings, D. Q. Mayne, Model Predictive Control: Theory and Design, Nob Hill Publishing, Madison, WI, 2009.

[18] M. V. Kothare, V. Balakrishnan, M. Morari, Robust constrained model predictive control using linear matrix inequalities, Automatica 32 (10) (1996) 1361-1379. 